\title{
AVALIAÇÃO ECOTOXICOLÓGICA DO RESÍDUO DE MINERAÇÃO DE CARVÃO
}

\author{
Luís Carlos Iuñes de Oliveira Filho(1)*, Dilmar Baretta(2), Talyta Zortéa(3), Katiane \\ Casarotto(2), Priscilla Dors(1), Mari Lucia Campos(4) e Júlio Cesar Pires Santos(4) $^{(4)}$
}

(1) Universidade do Estado de Santa Catarina, Centro de Ciências Agroveterinárias, Departamento de Solos e Recursos Naturais, Programa de Pós-graduação em Ciência do Solo, Lages, Santa Catarina, Brasil.

(2) Universidade do Estado de Santa Catarina, Centro de Educação Superior do Oeste, Departamento de Zootecnia, Chapecó, Santa Catarina, Brasil.

(3) Universidade do Estado de Santa Catarina, Centro de Educação Superior do Oeste, Departamento de Zootecnia, Programa de Pós-graduação em Zootecnia, Chapecó, Santa Catarina, Brasil.

(4) Universidade do Estado de Santa Catarina, Centro de Ciências Agroveterinárias, Departamento de Solos e Recursos Naturais, Lages, Santa Catarina, Brasil.

* Autor correspondente.

E-mail: iunes1981@gmail.com

\section{RESUMO}

O descarte de grandes quantidades de resíduos de mineração de carvão modifica quimicamente o solo e isso pode alterar a estrutura e atividade da fauna edáfica. Objetivou-se estudar os efeitos ecotoxicológicos das deposições de resíduo piritoso (carvão mais a pirita) em dois solos, coletados em Santa Catarina, Argissolo Vermelho-Amarelo e Nitossolo Háplico, nas doses de 0; 2,5; 5; 10 e 20 \% desse resíduo. Os testes realizados foram sobrevivência e reprodução de Enchytraeus crypticus e de germinação com Avena sativa e Lotus corniculatus. $O$ valor de $\mathrm{pH}$, independentemente do tipo do solo, diminuiu à medida que aumentou a dose de resíduo piritoso. $O$ resíduo piritoso na menor dose resultou em efeitos negativos ao $E$. crypticus, como redução na taxa sobrevivência de adultos e nenhuma reprodução. Nos testes de sobrevivência e reprodução de enquitreídeos, o valor de $\mathrm{LC}_{50}$ (concentração estimada que pode causar efeitos negativos na sobrevivência e germinação em $50 \%$ de um grupo de organismos) ficou abaixo $4 \%$ e $^{-E_{50}}$ (efeitos negativos na reprodução) $<2,5 \%$ para os dois solos, respectivamente. A exposição de $A$. sativa aos dois solos não expressou efeito tóxico para $L$. corniculatus, no Nitossolo. Porém, no Argissolo, 


\begin{abstract}
a contaminação causou efeitos na menor dose para $L$. corniculatus. No teste de germinação de sementes, somente $L$. corniculatus apresentou sensibilidade no Argissolo Vermelho-Amarelo $\left(\mathrm{LC}_{50}<4 \%\right.$ ). A elevada sensibilidade dos organismos do solo avaliado à aplicação do resíduo piritoso influenciou-os negativamente, tanto em um curto período de tempo quanto em longo prazo.
\end{abstract}

\title{
Palavras-chave: resíduo piritoso, Enchytraeus crypticus, Avena sativa, Lotus corniculatus.
}

\begin{abstract}
ECOTOXICOLOGICAL ASSESSMENT OF COAL MINING WASTE
The disposal of large amounts of coal mining waste chemically modifies the soil, and this may change the structure and activity of soil fauna. The objective of this investigation was to study the ecotoxicological effects of depositions of pyritic waste (coal plus pyrite) in two soils collected in Santa Catarina, Argissolo Vermelho-Amarelo (Ultisol) e Nitossolo Háplico (Hapludox), at the application rates of 0,2.5, 5, 10, and $20 \%$ of that residue. Survival and reproduction of Enchytraeus crypticus and germination of Avena sativa and Lotus corniculatus were tested. The $\mathrm{pH}$ value, regardless of the type of soil, decreased as the rate of pyritic waste increased. Pyritic waste at the lowest rate resulted in negative effects on E. crypticus, as shown by reduction in the adult survival rate and no reproduction. In survival and reproduction testing of enchytraeids, the value of $L_{50}$ (estimated concentration that can cause negative effects on the survival and germination of $50 \%$ of a group of organisms) was below $4 \%$, and $E C_{50}$ (negative effects on reproduction) was less than $2.5 \%$ for both soils. Exposure of A. sativa to the two soils did not have a toxic effect, nor did exposure of L. corniculatus to Hapludox. However, in the Ultisol, contamination caused effects at the lowest rate for L. corniculatus. In the seed germination test, only L. corniculatus showed sensitivity in the Ultisol $\left(L C_{50}<4 \%\right)$. High sensitivity to the application of pyritic residue adversely affects soil organisms, both in the short term and long term.
\end{abstract}

Keywords: pyritic waste, Enchytraeus crypticus, Avena sativa, Lotus corniculatus.

\section{INTRODUÇÃO}

A mineração de carvão é uma das principais atividades econômicas da região sul de Santa Catarina (Francisconi et al., 2013). Entretanto, essa atividade gera grande quantidade de rochas residuais e resíduos que ficam depositados na superfície (Chiochetta et al., 2013). Isso faz com que materiais de camadas subsuperficiais contendo pirita (sulfeto de ferro) sejam expostos a condições ambientais favoráveis a sua oxidação (Inda et al., 2010). Como consequência, ocorre diminuição de disponibilidade de nutrientes, aumentando a concentração de metais e a susceptibilidade dos solos à erosão, dificultando ou impedindo o desenvolvimento da vegetação (Albuquerque et al., 2011).

O conhecimento dos problemas causados pela oxidação da pirita é fundamental como subsídio para melhor definição de esquemas de construção do solocomo também da deposição do solo contendo pirita. Muitos estudos têm enfocado o efeito dos solos contaminados com pirita (Pereira et al., 2006; Alvarenga et al., 2012; Chiochetta et al., 2013); entretanto, são praticamente inexistentes as informações em relação ao efeito da adição de pirita sobre a biota.

A avaliação da contaminação dessas áreas tem sido realizada principalmente com o auxílio de parâmetros químicos (Terekhova, 2011). Todavia, essa informação não é suficiente para prever as alterações estruturais e funcionais na comunidade da biota do solo. Por isso, é importante considerar diferentes níveis tróficos para avaliar o risco de exposição ao solo contaminado.

A avaliação estrutural e a funcional medirão a capacidade do solo para ser usado para fins agrícolas, utilizando testes ecotoxicológicos para avaliar a contaminação e, ou, qualidade do solo. Os efeitos adversos sobre o solo incluem consequências sobre suas funções, principalmente como substrato vegetal e como hábitat para organismos edáficos (Loureiro et al., 2006).

A manutenção e conservação dos enquitreídeos, por exemplo, Enchytraeus crypticus, é de extrema importância, visto que esses organismos desempenham importante papel na decomposição da matéria orgânica e bioturbação em diferentes tipos 
de solo (Castro-Ferreira et al., 2012), ganhando cada vez mais atenção em estudos ecotoxicológicos do solo. Os testes com plantas, como Poaceae e Fabaceae, são considerados como ferramentas para identificar o efeito dos poluentes presentes nos solos, bem como para verificar o sucesso de processos de reparação de solos contaminados (Loureiro et al., 2006).

A região sul catarinense é uma das áreas que apresentam um dos maiores passivos ambientais do Brasil (Castilhos e Fernandes, 2011). Os recursos hídricos, o solo e o ar são os principais compartimentos que devem ser revistos para avaliar o risco ambiental e o humano. Nesse contexto, o objetivo deste estudo foi obter informações sobre o efeito da adição de resíduo piritoso gerado da mineração de carvão sobre invertebrados terrestres e plantas, com a finalidade de prever o impacto biológico da deposição desse material no solo.

\section{MATERIAL E MÉTODOS}

\section{Resíduo, solo e tratamentos}

O resíduo piritoso, neste estudo considerado como pirita mais carvão mineral, foi coletado em um "lavatório" de carvão no município de Lauro

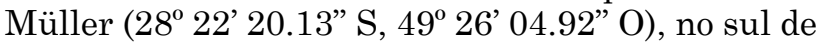
Santa Catarina, em 2012, o qual estava estocado em pilhas de 2-3 m por três anos. Os resíduos de carvão em toda região sul de Santa Catarina foram estudados por Silva et al. (2011), que verificaram maior abundância da pirita entre os Fe-minerais presentes. Foram feitas 10 amostragens nas pilhas, cada uma contendo em média $2 \mathrm{~kg}$ do resíduo piritoso. Aproximadamente sete dias antes de iniciar os testes, as amostras foram trituradas e passadas em peneira de malha $1,25 \mathrm{~mm}$ para maior homogeneização e armazenadas em recipientes hermeticamente fechados para reduzir o processo de oxidação do material.

Foram utilizados dois solos sem histórico de uso agrícola e de contaminação de pirita, um ArgissoloVermelho-Amarelo (PVA), em Lauro Müller, distante aproximadamente $4 \mathrm{~km}$ do local onde foi coletado o resíduo, e um Nitossolo Háplico (NX), em Capão Alto na Serra catarinense. O PVA foi coletado na borda de um remanescente de mata $\left(28^{\circ} 23^{\prime} 28.40^{\prime \prime} \mathrm{S}, 49^{\circ} 28^{\prime} 12.16^{\prime \prime} \mathrm{O}\right)$ e o NX em área coberta com gramíneas nativas à beira da rodovia SC-458 ( $\left.27^{\circ} 55^{\prime} 48.63^{\prime \prime} \mathrm{S}, 50^{\circ} 31^{\prime} 55.97^{\prime \prime} \mathrm{O}\right)$ ), ambos na camada de 0,00-0,10 m. Todos os solos foram previamente peneirados (malha de $4 \mathrm{~mm}$ ) e defaunados (com dois ciclos de congelamento-descongelamento, 24/24 h). A caracterização dos solos e a do resíduo são apresentadas no quadro 1.

Os tratamentos consistiram dos solos PVA e NX sem aplicação de resíduo (dose 0) e de combinações dos solos mais o resíduo, nas doses: 2,$5 ; 5 ; 10$; e $20 \%$, equivalendo a $25.641,52.632,111.111$ e $250.000 \mathrm{~kg} \mathrm{ha}^{-1}$, respectivamente, considerando $0,10 \mathrm{~m}$ de profundidade. Como na literatura não foi encontrado nenhum trabalho com doses de resíduo piritoso e nenhum que tenha estimado quantidades no solo, decidiu-se pela menor dose em virtude de se ter uma mistura o mais

Quadro 1. Caracterização física e química do Argissolo Vermelho-Amarelo (PVA), Nitossolo Háplico (NX) e do resíduo piritoso utilizado nos testes

\begin{tabular}{|c|c|c|c|c|c|}
\hline Atributo & PVA & NX & Resíduo piritoso & $\operatorname{LOD}^{(8)}$ & $\mathrm{LOQ}^{(9)}$ \\
\hline $\mathrm{pH}(\mathrm{KCl})(1)$ & 3,55 & 4,48 & ND & & \\
\hline $\operatorname{MOS}(\%)^{(2)}$ & 4,7 & 1,5 & ND & & \\
\hline CTC pH 7,0 $\left(\mathrm{cmol}_{\mathrm{c}} \mathrm{dm}^{-3}\right)^{(3)}$ & 45,39 & 35,23 & ND & & \\
\hline $\mathrm{Al}^{3+}\left(\mathrm{cmol}_{\mathrm{c}} \mathrm{dm}^{-3}\right)^{(4)}$ & 5,76 & 4,11 & ND & & \\
\hline $\mathrm{H}+\mathrm{Al}\left(\mathrm{cmol}_{\mathrm{c}} \mathrm{dm}^{-3}\right)^{(5)}$ & 43,30 & 30,70 & ND & & \\
\hline Argila $\left(\mathrm{g} \mathrm{kg}^{-1}\right)^{(6)}$ & $200(20 \%)$ & $160(16 \%)$ & ND & & \\
\hline Silte $\left(\mathrm{g} \mathrm{kg}^{-1}\right)^{(6)}$ & $500(50 \%)$ & $370(37 \%)$ & ND & & \\
\hline Areia $\left(\mathrm{g} \mathrm{kg}^{-1}\right)^{(6)}$ & $300(30 \%)$ & $470(47 \%)$ & ND & & \\
\hline \multicolumn{6}{|l|}{ Total(7) } \\
\hline $\mathrm{Cu}\left(\mathrm{mg} \mathrm{kg}{ }^{-1}\right)$ & $108 \pm 1,58$ & $26 \pm 0,56$ & $70 \pm 29,50$ & 0,01 & 0,05 \\
\hline $\mathrm{Zn}\left(\mathrm{mg} \mathrm{kg}^{-1}\right)$ & $126 \pm 11,93$ & $37 \pm 0,35$ & $59 \pm 15,10$ & 0,01 & 0,04 \\
\hline $\mathrm{Fe}(\mathrm{mg} \mathrm{kg}-1)$ & $406.711 \pm 37.090$ & $2.602 \pm 33,67$ & $106.127 \pm 87.157$ & 0,06 & 0,20 \\
\hline $\operatorname{Mn}\left(\mathrm{mg} \mathrm{kg}^{-1}\right)$ & $3.179 \pm 24,29$ & $14.016 \pm 499$ & $903 \pm 1.061$ & 0,37 & 1,22 \\
\hline
\end{tabular}

(1) $\mathrm{pH}$ em solução de $\mathrm{KCl} 1 \mathrm{~mol} \mathrm{~L}{ }^{-1}$, relação 1:5 m/v (ISO - International Organization for Standardization, 2004). (2) MOS: matéria orgânica do solo, oxidada com dicromato de potássio, em meio ácido (Tedesco et al., 1995). (3) CTC: capacidade de troca de cátions (Tedesco et al., 1995). (4) $\mathrm{Al}^{3+}$ : determinado por titulação ácido-base, extraído com $\mathrm{KCl} 1 \mathrm{~mol} \mathrm{~L}-1$ (Tedesco et al., 1995). (5) $\mathrm{H}+\mathrm{Al}$ (Tedesco et al., 1995). (6) Método da pipeta (Gee e Bauder, 1986). (7) Solo: digestão em aqua regia (Soares, 1995); Resíduo piritoso: digestão em forno micro-ondas com $4 \mathrm{~mL}$ de $\mathrm{HNO}_{3}$ e $3 \mathrm{~mL} \mathrm{HCl}$ (USEPA, 2007). ND: valor não determinado. (8) LOD: limite de detecção operacional. (9) LOQ: limite de quantificação operacional do EAA-FG CONTRAA 700 (AnalytikJena). O valor após o símbolo \pm representa o desvio-padrão da média $(n=5)$. 
homogênea possível, visto que a quantidade do resíduo a ser misturado ao solo foi muito pequena. É importante ressaltar que essa contaminação incorporada retrata uma situação hipotética de contaminação. Caso o resíduo fosse aplicado somente em superfície, os resultados poderiam apresentar falso-negativos, especialmente se os organismos ficassem abaixo da superfície sem ter contato com o resíduo e tempo hábil para oxidá-lo, no caso do teste de germinação.

\section{Parâmetros gerais dos testes}

O substrato utilizado para validar os testes foi um solo artificial, em que a formulação original foi modificada, com utilização da fibra de coco no lugar da turfa, diminuindo seu conteúdo para $5 \%$, denominado de solo artificial tropical (SAT) (Kuperman et al., 2009). A fibra de coco foi utilizada em virtude de ser um material barato, de fácil obtenção e maior disponibilidade. Para os testes de sobrevivência e reprodução de enquitreídeos e de germinação, foram montadas cinco réplicas inteiramente casualizadas, como descrito mais adiante. Todas as réplicas permaneceram em câmara com temperatura controlada a $20{ }^{\circ} \mathrm{C}$ para enquitreídeos e $22^{\circ} \mathrm{C}$ para germinação, com fotoperíodo de 16:8 h (luz:escuro) e pH e umidade determinados no início e no final do teste. Previamente, o solo foi umedecido a $60 \%$ da capacidade de retenção de água (CRA) para enquitreídeos e avaliação do $\mathrm{pH}$ e a $70 \%$ da CRA para germinação. Os espécimes de E. crypticus usados no teste foram obtidos de culturas mantidas em laboratório, de acordo com o protocolo da ISO 16387 (ISO, 2004). As sementes utilizadas nos testes foram obtidas de fonte comercial.

\section{Avalição do pH}

Foi montado um teste para avaliar o $\mathrm{pH}$ em $\mathrm{KCl}$ (ISO, 2004) com cinco réplicas de cada tratamento, onde no início (dia zero) e final de sete e 28 dias foram avaliados, como forma de obter um controle maior sobre a variação desse atributo. Esse teste foi conduzido em recipiente $(0,05 \mathrm{~m}$ de diâmetro e $0,07 \mathrm{~m}$ altura) preenchido com $0,1 \mathrm{~kg}$ de solo fresco, com temperatura, umidade e fotoperíodo controlados, sem organismos.

\section{Teste de sobrevivência e reprodução com Enchytraeus crypticus}

O teste que determina a reprodução de Enchytraeus crypticus seguiu o protocolo padronizado da ISO 16387 (ISO, 2004). Por causa do pequeno tamanho e curto ciclo reprodutivo dessa espécie, a duração do teste foi reduzida para 28 dias, e os adultos foram mantidos nos recipientes por esse período (Chelinho et al., 2011). Dez adultos com o clitelo desenvolvido foram introduzidos em cada recipiente (diâmetro: 0,03 m; altura: $0,05 \mathrm{~m}$ ), contendo $23 \mathrm{~g}$ de solo fresco mais o suprimento de comida $(50 \mathrm{mg}$ de flocos de aveia moídos e autoclavados, sendo fornecido $25 \mathrm{mg}$ de comida aos 7, 14 e 21 dias). Ao final do teste, o número de enquitreídeos foi avaliado após fixação com álcool
(80 \%), colorindo com Rosa Bengala (solução de $1 \%$ com etanol) e peneiragem úmida (malha $103 \mathrm{~mm}$ ).

\section{Teste de germinação com Avena sativa e Lotus corniculatus}

O teste de germinação foi com base no protocolo OECD 208 (Organization for Economic Co-operation and Development, 2006), utilizando as espécies Avena sativa (Poaceae) e Lotus corniculatus (Fabaceae), com cinco réplicas por tratamento, onde cada réplica consistiu de uma placa de petri $(0,10 \mathrm{~m}$ de diâmetro e $0,02 \mathrm{~m}$ de altura), preenchida com $0,1 \mathrm{~kg}$ de solo fresco e 10 sementes por réplica. Após sete dias, o número de sementes germinadas foi contabilizado.

\section{Análise estatística}

Para os testes de sobrevivência e reprodução, germinação e evolução do $\mathrm{pH}$ em $\mathrm{KCl}$, diferenças entre o solo contaminado e o de referência foram avaliadas pela análise de variância (One-way Anova) seguida pelo teste de Dunnett $(\mathrm{M} \leq$ controle, $\mathrm{p}<0,05)$, utilizando o software Statistica 7.0 (Statsoft Inc., Tulsa, EUA).

Valores de $\mathrm{LC}_{50}$ (concentração estimada na qual se espera causar letalidade em $50 \%$ de um grupo de organismos, efeitos negativos na sobrevivência e germinação) foram calculados, utilizando o software PriProbit ${ }^{\circledR} 1.63$ (Graduate School of Agriculture, Kyoto, Japão). Para o teste de reprodução, os efeitos de $\mathrm{EC}_{50}$ (efeitos negativos na reprodução) não puderam ser realizados, visto que não houve reprodução de enquitreídeos.

\section{RESULTADOS E DISCUSSÃO}

\section{Características do solo}

De acordo com os valores orientadores de prevenção (VP) estabelecidos pela Companhia Ambiental do Estado de São Paulo (Cetesb, 2014), em nenhuma dose os teores de $\mathrm{Cu}$ e $\mathrm{Zn}$ estimados aproximam-se do limite de VP $\left(60 \mathrm{mg} \mathrm{kg}^{-1} \mathrm{de} \mathrm{Cu}\right.$ e $86 \mathrm{mg} \mathrm{kg}^{-1}$ de Zn) (Quadro 2). Para Fe e Mn, não foram estabelecidos valores orientadores para o solo.

Quadro 2. Valores de metais estimados para cada dose de resíduo piritoso $(0 ; 2,5 ; 5 ; 10 ;$ e $20 \%)$, independentemente do tipo de solo

\begin{tabular}{lcccc}
\hline \multirow{2}{*}{ Metal } & \multicolumn{4}{c}{ Dose do resíduo piritoso } \\
\cline { 2 - 5 } & $\mathbf{2 , 5} \%$ & $\mathbf{5 \%}$ & $\mathbf{1 0} \%$ & $\mathbf{2 0} \%$ \\
\hline Cu (mg kg-1 de solo) & 1,8 & 3,7 & 7,8 & 17,5 \\
Zn (mg kg-1 de solo) & 1,5 & 3,1 & 6,6 & 14,8 \\
Fe (mg kg-1 de solo) & 2.721 & 5.586 & 11.792 & 26.532 \\
Mn (mg kg-1 de solo) & 23,2 & 47,5 & 100,0 & 226,0 \\
\hline
\end{tabular}


Ambos os solos-controle (PVA 0 e NX 0) apresentaram valor de $\mathrm{pH}$ muito baixo. $\mathrm{O}$ valor de $\mathrm{pH}$ para os dois solos testados foi diferente entre as doses de resíduo aplicadas, em comparação ao seu respectivo controle (PVA 0 e NX 0) nas três datas avaliadas $(p<0,001)$ (Quadro 3). De maneira geral, o $\mathrm{pH}$ em $\mathrm{KCl}$, independentemente do tipo de solo estudado, diminuiu à medida que aumentou a dose de resíduo piritoso adicionada, especialmente quando comparada às doses $(2,5 ; 5 ; 10 ;$ e $20 \%)$ com o controle (0).

A diminuição do valor de $\mathrm{pH}$ entre as doses indicou que houve oxidação da pirita (Quadro 3). Quando os resíduos de pirita entram em contato com a umidade e o oxigênio, resultam na oxidação de sulfuretos, com queda acentuada no $\mathrm{pH}$ e na solubilização de contaminantes (metais), que se infiltraram no solo, elevando o nível de poluição (Martin Peinado et al., 2015). A oxidação da pirita é acelerada pela exposição do resíduo na superfície do solo, porque se trata de um processo aeróbico (Moses et al., 1987). Assim, áreas que passam por processos de recuperação e que tenham resíduos piritosos de carvão em superfície podem apresentar diminuição do $\mathrm{pH}$ mais acentuada e mais rápida.

\section{Validação dos testes}

Ambos os testes alcançaram os critérios de validade, de acordo com o seu respectivo protocolo.

\section{Impacto nos enquitreídeos}

Em solos contaminados, a menor dose ocasionou redução na taxa de sobrevivência de adultos de enquitreídeos no PVA ( $\mathrm{F}=5,98 ; \mathrm{p}<0,001)$ e NX $(\mathrm{F}=5,88 ; \mathrm{p}<0,001)$. Valores estimados de $\mathrm{LC}_{50}$ foram 3,40\% (34.872 $\left.\mathrm{kg} \mathrm{ha}^{-1}\right)$ no PVA e 3,85\% (39.487 kg ha-1) no NX; nesse caso, o intervalo de confiança não pode ser calculado. A reprodução dos enquitreídeos foi influenciada pela dose de resíduo no PVA ( $\mathrm{F}=16,94 ; \mathrm{p}<0,00001)$ e no NX ( $\mathrm{F}=387,94$; $\mathrm{p}<0,0001$ ), em todas as doses avaliadas do resíduo nos dois solos não houve reprodução (Figura 1), sendo o valor de $\mathrm{EC}_{50}<2,5 \%$; nesse caso, o intervalo de confiança não pode ser calculado. Embora o número de juvenis nos solos não contaminados (PVA 0 e NX 0) tenha ficado abaixo do critério de validade ( $15 \pm 8$ e $23 \pm 3$, respectivamente para o PVA e o NX), isso não inviabilizou o teste, já que esse é aplicado ao solo-referência, que nesse caso é o SAT.

$\mathrm{O}$ valor de $\mathrm{pH}$ natural dos solos pode ter sido um dos fatores que influenciou a baixa reprodução de enquitreídeos nos solos-controle (PVA 0 e NX 0); no entanto, parece não ter influenciado a sobrevivência deles (Figura 1). A exposição dos enquitreídeos ao resíduo piritoso (solos contaminados) ocasionou baixa sobrevivência e inibição na reprodução, a partir da menor dose (Figura 1). De maneira geral, nos testes crônicos são observados efeitos subletais, que permitem a sobrevivência, mas interferem em uma ou mais funções biológicas, como inibição do crescimento, reprodução e alterações morfológicas. Baixo número de juvenis foi encontrado em valores de $\mathrm{pH} 4,8$ e quase nenhum juvenil com $\mathrm{pH}$ inferior a 4,0 em trabalho conduzido por Jänsch et al. (2005). Também, o que pode ter influenciado são os teores naturais de metais nos dois solos (Quadro 1), podendo ser confirmado por Tosza et al. (2010), onde a abundância de enquitreídeos aumentou com a distância a partir da fonte da poluição, evidenciando um gradiente oposto ao das concentrações de metal.

Ambos os solos apresentaram elevados teores de $\mathrm{Mn}$, aproximadamente $3.100 \mathrm{mg} \mathrm{kg}^{-1}$ no PVA e $14.000 \mathrm{mg} \mathrm{kg}^{-1}$ no NX (Quadro 1). De acordo com Kuperman et al. (2004), valores de $\mathrm{EC}_{50}$ de 192 (147-238) $\mathrm{mg} \mathrm{kg}^{-1}$ diminuiu a reprodução de juvenis, e com 644 mg kg-1 não ocorreu reprodução (O PVA

Quadro 3. Valores médios de pH em KCl do Argissolo Vermelho-Amarelo (PVA) e Nitossolo Háplico (NX), ao final de sete e 28 dias

\begin{tabular}{lcllc}
\hline Solo & Dose de resíduo piritoso (\%) & Dia zero & 7 dias após & 28 dias após \\
\hline PVA & 0 & $3,55 \pm 0,03$ & $3,52 \pm 0,01$ & $3,90 \pm 0,02$ \\
PVA & 2,5 & $3,32 \pm 0,02^{*}$ & $3,28 \pm 0,01^{*}$ & $3,40 \pm 0,01^{*}$ \\
PVA & 5 & $3,16 \pm 0,02^{*}$ & $3,09 \pm 0,01^{*}$ & $3,11 \pm 0,04^{*}$ \\
PVA & 10 & $2,93 \pm 0,01^{*}$ & $2,85 \pm 0,01^{*}$ & $2,83 \pm 0,15^{*}$ \\
PVA & 20 & $2,54 \pm 0,02^{*}$ & $2,52 \pm 0,01^{*}$ & $2,56 \pm 0,09^{*}$ \\
NX & 0 & $4,48 \pm 0,10$ & $4,57 \pm 0,01$ & $4,57 \pm 0,02$ \\
NX & 2,5 & $4,29 \pm 0,05^{*}$ & $4,29 \pm 0,01^{*}$ & $4,15 \pm 0,01^{*}$ \\
NX & 5 & $4,00 \pm 0,02^{*}$ & $4,06 \pm 0,01^{*}$ & $4,10 \pm 0,01^{*}$ \\
NX & 10 & $3,59 \pm 0,02^{*}$ & $3,70 \pm 0,01^{*}$ & $3,81 \pm 0,01^{*}$ \\
NX & 20 & $3,02 \pm 0,02^{*}$ & $3,21 \pm 0,01^{*}$ & $3,33 \pm 0,06^{*}$ \\
\hline
\end{tabular}

Asterisco $\left(^{*}\right)$ na coluna difere do respectivo controle (PVA 0 e NX 0) pelo teste de Dunnett $(\mathrm{M} \leq$ controle). Doses: $0=$ controle $($ sem resíduo); $2,5=2,5 \%$ de resíduo; $5=5 \%$ de resíduo; $10=10 \%$ de resíduo; $20=20 \%$ de resíduo. $\mathrm{O}$ valor após o símbolo \pm representa o desvio-padrão da média $(n=5)$. 


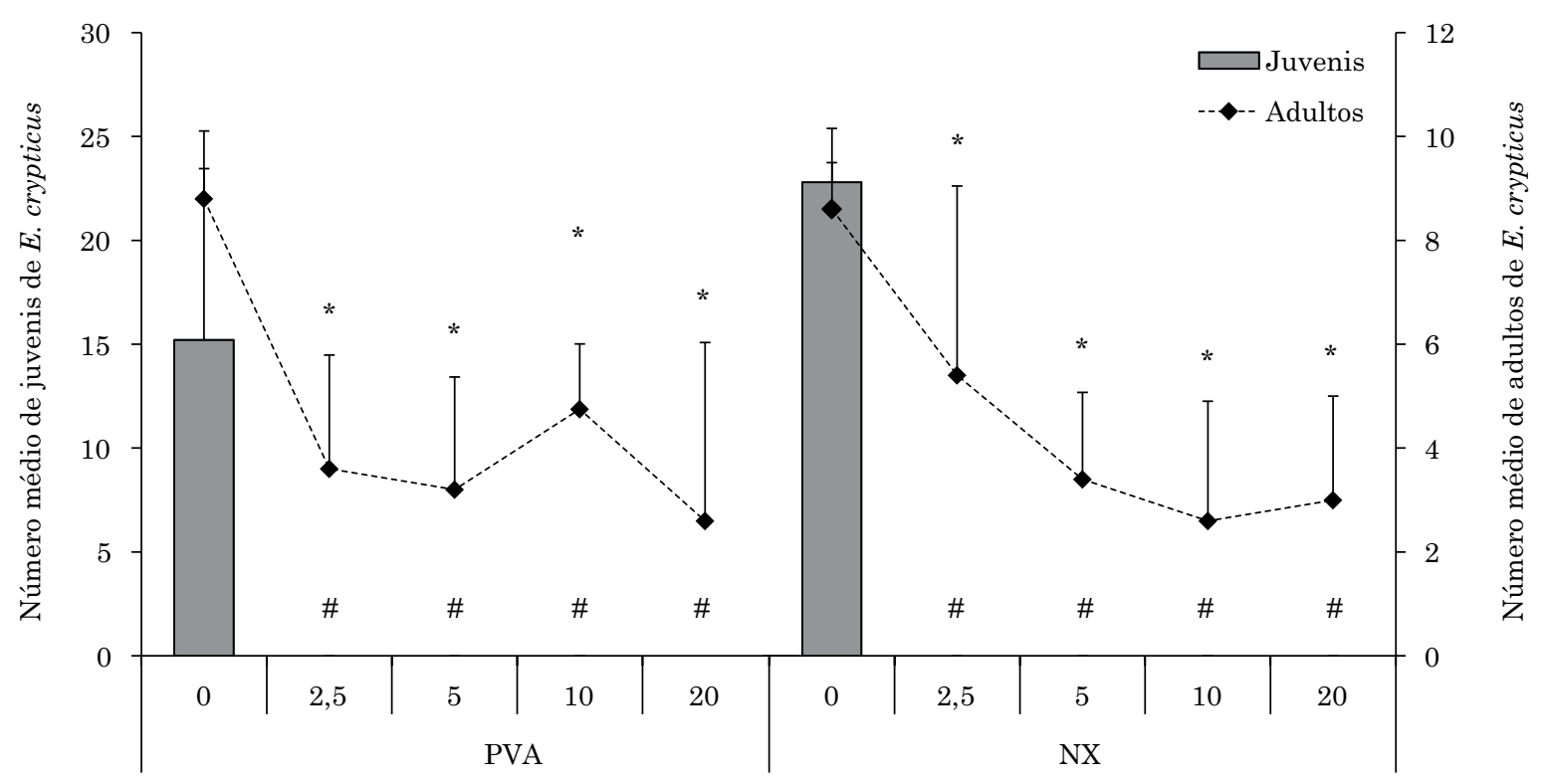

Figura 1. Sobrevivência e reprodução de Enchytraeus crypticus quando exposto a solo não contaminado e contaminado. $(T)$ desvio-padrão $(n=5)$. \# $\mathrm{e}$ *: significativo para número médio de juvenis e número médio de adultos, $\mathbf{p} \leq \mathbf{0 , 0 5}$, respectivamente, avaliado pelo teste de Dunnett. PVA: Argissolo Vermelho-Amarelo; NX: Nitossolo Háplico; 0 = controle (sem resíduo); 2,5 = 2,5 \% de resíduo; $5=5 \%$ de resíduo; $10=10 \%$ de resíduo; e $20=20 \%$ de resíduo.

apresentou valores mais elevados de $\mathrm{Cu}$ e $\mathrm{Zn}$ que o NX (Quadro 1) e maiores que o VP (60 $\mathrm{mg} \mathrm{kg}^{-1} \mathrm{de}$ $\mathrm{Cu}$ e $86 \mathrm{mg} \mathrm{kg}-1$ de $\mathrm{Zn}$ ) proposto pela Cetesb (2014). Esse fato pode ter influenciado a menor reprodução no PVA não contaminado.

Em solos tropicas contaminados com $\mathrm{Cu}$, Konečný et al. (2014) encontraram valores de $\mathrm{EC}_{50}$ de $351 \mathrm{mg} \mathrm{kg}{ }^{-1}$, causando redução no número de juvenis de E. crypticus. Em solo natural contaminado artificialmente, Jensen e Pedersen (2005) encontraram para $\mathrm{Zn}$ valores de $\mathrm{EC}_{50}$ de $262 \mathrm{mg} \mathrm{kg}-1$, que foram suficientes para ocasionar diminuição na reprodução de $E$. crypticus. Nesse estudo, os valores de $\mathrm{Cu}$ e $\mathrm{Zn}$ no solo contaminado não se aproximaram desses valores.

\section{Impactos na germinação}

A exposição de A. sativa aos dois solos não expressou efeito tóxico (PVA: $\mathrm{F}=0,64 ; \mathrm{p}=0,63$; NX: $F=0,96 ; p=0,45)$ para $L$. corniculatus no NX $(\mathrm{F}=0,80 ; \mathrm{p}=0,54)$ com valor estimado de $\mathrm{LC}_{50}>20 \%$ (250.000 kg ha-1). Porém, para L. corniculatus, a exposição ao PVA contaminado após sete dias de exposição (Figura 2) causou efeitos $(\mathrm{F}=34,06$; $\mathrm{p}<0,0001)$ em todas as doses, com valor estimado de $\mathrm{LC}_{50}$ de $3,48 \%$ (35.692 $\left.\mathrm{kg} \mathrm{ha}^{-1}\right)$.

Extensa avaliação sobre os efeitos da acidificação do solo em diversas espécies de plantas foi realizada por Azevedo et al. (2013) que constataram que a ocorrência preferencial quanto ao $\mathrm{pH}$ para $A$. sativa está entre 6,2 e 9,2; e para $L$. corniculatus, entre 4,2 e 5,9. Embora o pH nos dois solos, após sete dias de germinação, tenha ficado abaixo do preferencial (Quadro 3), esse não apresentou nenhum efeito na germinação de sementes para A. sativa. (Figura 2). Loureiro et al. (2006) também verificaram que A. sativa mal refletia o potencial de fitotoxicidade de solos com baixo $\mathrm{pH}$ e contaminado por metais. Para L corniculatus, a germinação de sementes foi influenciada somente no PVA (Figura 2). A toxicidade não depende somente de concentrações específicas; a forma química do metal, a presença de outros produtos químicos que possam agravar ou melhorar a toxicidade do metal, o teor de nutrientes e o $\mathrm{pH}$, que prevalece no solo contaminado, interferirão o modo de como as plantas respondem ao metal (Freitas et al., 2004). O baixo valor de $\mathrm{pH}$ pode ter comprometido a germinação em razão da presença de metais como $\mathrm{Al}^{3+} \mathrm{e} \mathrm{Mn}^{2+}$ (Moses et al., 1987).

O que pode explicar a baixa fitotoxicidade nos solos contaminados neste estudo é que a utilização da capacidade de germinação como variável resposta tem sido relatada com baixa sensibilidade do que os outros parâmetros, como o crescimento inicial das plântulas (Loureiro et al., 2006). É essencial escolher plantas tolerantes aos metais específicos, bem como tolerância à seca e falta de nutrientes frequentes em áreas de mineração. Também, o plantio de diferentes espécies de gramíneas, como A. sativa; leguminosas, como L. corniculatus; e a rotação com espécies nativas serão capazes de restaurar a 


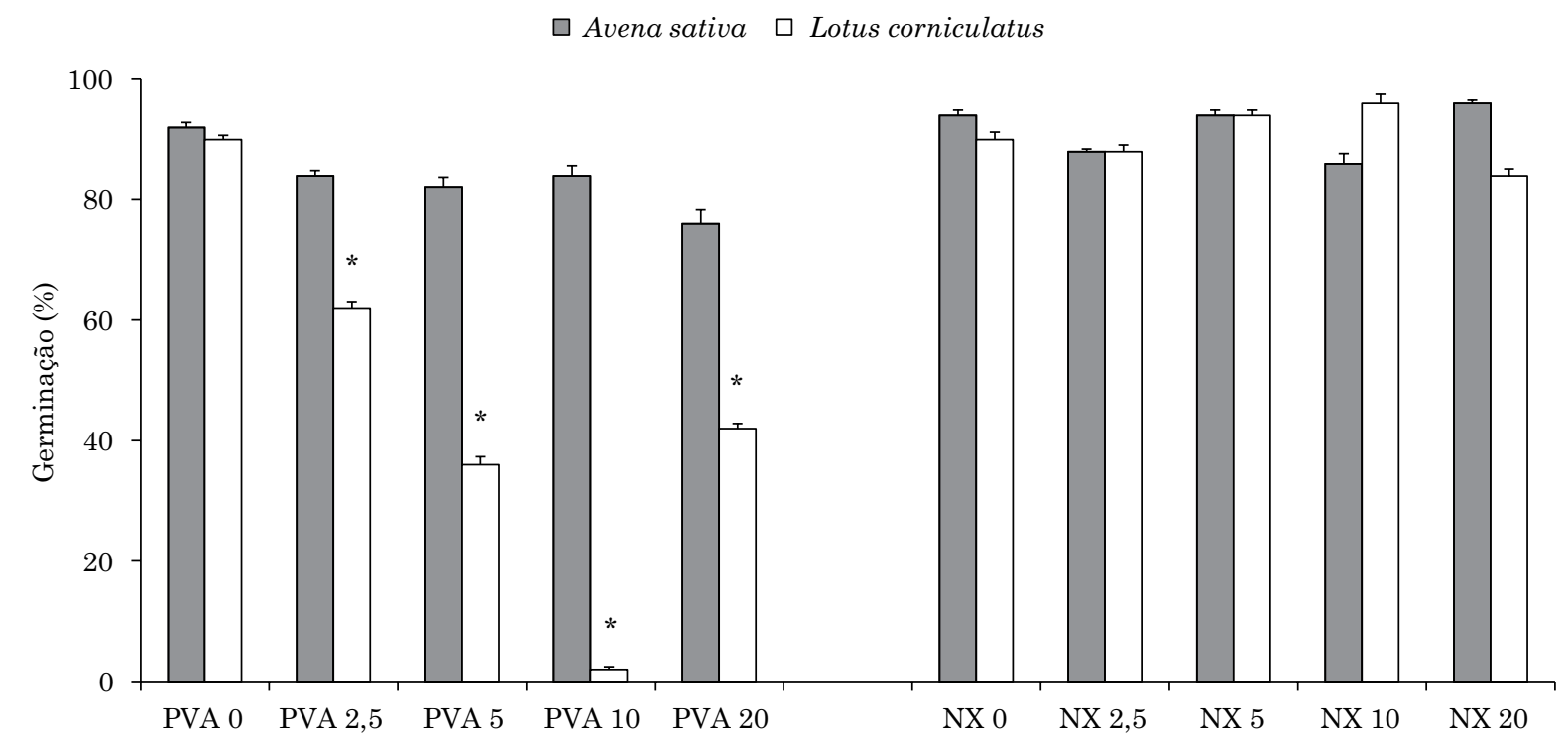

Figura 2. Germinação de Avena sativa e Lotus corniculatus quando expostas a solo não contaminado e contaminado. $(T)$ desvio-padrão $(n=5)$. * significativo, $p \leq 0,05$, avaliado pelo teste de Dunnett, em relação a sua respectiva referência. PVA: Argissolo Vermelho-Amarelo; NX: Nitossolo Háplico; 0 = controle (sem resíduo); $2,5=2,5 \%$ de resíduo; $5=5 \%$ de resíduo; $10=10 \%$ de resíduo; e $20=20 \%$ de resíduo.

fertilidade do solo e acelerar a sucessão ecológica (Freitas et al., 2004).

Com a diminuição do pH (Quadro 3), a biodisponibilidade dos metais decorrentes do resíduo piritoso potencialmente pode estar aumentando de acordo com a dose (Quadro 2), visto que o baixo pH está geralmente associado à elevada biodisponibilidade de produtos químicos e, consequentemente, à toxicidade elevada (Phillips et al., 2002). A adição do resíduo piritoso ao solo ocasionou a diminuição do $\mathrm{pH}$ e isso pode causar efeitos diretos pela acidificação do solo e indiretos pela biodisponibilidade dos metais, como $\mathrm{Cu}, \mathrm{Zn}$ e $\mathrm{Mn}$, presentes no solo e no resíduo, e consequente toxicidade para plantas e animais. De fato, é difícil discriminar entre efeitos diretos e indiretos do $\mathrm{pH}$ que estão influenciando o desenvolvimento das plantas, nesse caso, o baixo $\mathrm{pH}$, algum metal em específico ou mistura desses metais.

Embora a sobrevivência e a reprodução de enquitreídeos tenham sido mais influenciadas que a germinação (Figuras 1 e 2), deve-se tomar cuidado na interpretação dos resultados, pois o período de exposição ao resíduo piritoso foi curto em relação ao tempo e à quantidade que esse material pode estar causando danos ao ambiente. As diferentes sensibilidades das espécies demonstram a necessidade de incluir organismos representativos de diferentes níveis tróficos em uma bateria de testes, aumentando a relevância ecológica em diferentes solos, visto que os organismos têm diferentes vias de absorção.

\section{CONCLUSÕES}

Os organismos evidenciam sensibilidade elevada à aplicação do resíduo piritoso (carvão mais a pirita).

O resíduo piritoso influencia negativamente Enchytraeus crypticus e Lotus corniculatus, tanto em curto período de tempo (teste de germinação) quanto em longo prazo (reprodução).

As diferenças na sensibilidade de espécies podem ser um fator importante, pois Avena sativa não apresentou resposta negativa nos testes realizados, fazendo-se necessária a utilização de outros parâmetros, como crescimento e desenvolvimento.

\section{AGRADECIMENTOS}

À Coordenação de Aperfeiçoamento de Pessoal de Nível Superior (Capes), pela concessão de bolsa.

\section{REFERÊNCIAS}

Albuquerque JA, Almeida JA, Gatiboni LC, Eltz FLF. Atividades agrícolas de produção em solos frágeis no sul do Brasil. Tópicos Ci Solo. 2011;7:367-403.

Alvarenga P, Palma P, Varennes A, Cunha-Queda AC. A contribution towards the risk assessment of soils from the São Domingos Mine (Portugal): Chemical, microbial and ecotoxicological indicators. Environ Pollut. 2012;161:50-6. 
Azevedo LB, van Zelm R, Hendriks AJ, Bobbink R, Huijbregts MAJ. Global assessment of the effects of terrestrial acidification on plant species richness. Environ Pollut. 2013;174:10-5.

Castilhos ZC, Fernandes FRC. A bacia carbonífera sul catarinense e os impactos e passivos da atividade da indústria extrativa mineral de carvão na territorialidade. In: Fernandes FRC, Enríquez MARS, Alamino RCJ, editores. Recursos minerais e sustentabilidade territorial: grandes minas. Rio de Janeiro: CETEM/MCTI; 2011. p.361-86.

Castro-Ferreira MP, Roelofs D, van Gestel CAM, Verweij RA, Soares AMVM, Amorim MJB. Enchytraeus crypticus as model species in soil ecotoxicology. Chemosphere. 2012;87:1222-7.

Chelinho S, Domene X, Campana P, Natal-da-Luz T, Scheffczyk A, Römbke J, Andrés P, Sousa JP. Improving ecological risk assessment in the Mediterranean area: Selection of reference soils and evaluating the influence of soil properties on avoidance and reproduction of two oligochaete species. Environ Toxicol Chem. 2011;30:1050-8.

Chiochetta CG, Radetski MR, Corrêa AXR, Tischer V, Tiepo EN, Radetski CM. Abandoned coal mining sites: Using ecotoxicological tests to support an industrial organic sludge amendment. Environ Sci Pollut Res. 2013;20:7656-65.

Companhia Ambiental do Estado de São Paulo - Cetesb. Valores orientadores para solo e água subterrânea no estado de SP [internet]. São Paulo, SP: Cetesb; 2014. [Acesso em 1 mar 2014]. Disponível em: http://www.cetesb.sp.gov.br/userfiles/file/ institucional/do/2014/DD-045-2014-P53.pdf.

Francisconi MS, Bona A, Silveira FZ, Geremias R, Pich CT. Use of coal mining waste for river water treatment and evaluation of this process using physicochemical parameters and bioassays. Ecotoxicol Environ Contam. 2013;8:45-51.

Freitas H, Prasad MN V, Pratas J. Plant community tolerant to trace elements growing on the degraded soils of São Domingos mine in the south east of Portugal: Environmental implications. Environ Inter. 2004;30:65-72.

Gee GW, Bauder JW. Particle size analysis. In: Klute A, editor. Methods of soil analysis. Part 1 Physical and mineralogical methods. 2nd ed. Madison: American Society of Agronomy and Soil Science Society of America; 1986. p.383-411.

Inda AV, Quinõnes ORG, Giasson E, Bissani CA, Dick DP, Nascimento PC. Atributos químicos relacionados ao processo de sulfurização em solos construídos após mineração de carvão. Ci Rural. 2010;40:1060-7.

International Organization for Standardization - ISO. Soil quality - Effects of pollutants on Enchytraeidae (Enchytraeus sp.) - Determination of effects on reproduction and survival. ISO 16387. Geneva: 2004.

Jänsch S, Amorim MJ, Römbke J. Identification of the ecological requirements of important terrestrial ecotoxicological test species. Environ Rev. 2005;13:51-83.

Jensen J, Pedersen MB. Ecological risk assessment of contaminated soil. Rev Environ Contam Toxicol. 2005;186:73-105.

Konečný L, Ettler V, Kristiansen SM, Amorim MJB, Kříbek B, Mihaljevič M, Šebek O, Nyambe I., Scott-Fordsmand, JJ. Response of Enchytraeus crypticus worms to high metal levels in tropical soils polluted by copper smelting. J Geochem Explor. 2014;144:427-32.

Kuperman RG, Checkai RT, Garcia MVB, Römbke J, Stephenson GL, Sousa JP. State of the science and the way forward for the ecotoxicological assessment of contaminated land. Pesq Agropec Bras. 2009;44:811-24.

Kuperman RG, Checkai RT, Simini M, Phillips CT. Manganese toxicity in soil for Eisenia fetida, Enchytraeus crypticus (Oligochaeta), and Folsomia candida (Collembola). Ecotoxicol Environ Saf. 2004;57:48-53.

Loureiro S, Santos C, Pinto G, Costa A, Monteiro M, Nogueira AJA, Soares AM. Toxicity assessment of two soils from Jales mine (Portugal) using plants: Growth and biochemical parameters. Arch Environ Contam Toxicol. 2006;50:182-90.

Martin Peinado FJ, Romero-Freire A, Garcia Fernández I, Sierra Aragón M, Ortiz-Bernad I, Simón Torres M. Long-term contamination in a recovered area affected by a mining spill. Sci Total Environ. 2015;514:219-23.

Moses CO, Nordstrom DK, Herman JS, Mills AL. Aqueous pyrite oxidation by dissolved oxygen and by ferric iron. Geochim Cosmochim Acta. 1987;51:1561-71.

Organization for Economic Co-Operation and Development - OECD. Guidelines for testing of chemicals. Test no 208: Terrestrial plant test: seedling emergence and seedling growth test. Paris: OECD Publishing; 2006.

Pereira R, Sousa JP, Ribeiro R, Gonçalves F. Microbial indicators in mine soils (S. Domingos Mine, Portugal). Soil Sediment Contam. 2006;15:147-67.

Phillips CT, Kuperman RG, Checkai RT. Toxicity of chemical-warfare agent HD to Folsomia candida in different soil types. Eur J Soil Biol. 2002;38:281-5.

Silva LFO, Macias F, Oliveira MLS, Boit MK, Waanders F. Coal cleaning residues and Fe-minerals implications. Environ Monit Assess. 2011;172:367-78.

Soares ER. Mobilização de metais pesados em materiais provenientes do complexo carboenergético de Candiota-RS [dissertação]. Viçosa, MG: Universidade Federal de Viçosa; 1995.

Tedesco MJ, Gianello C, Bissani CA, Bohnen H, Volkweiss SJ. Análise de solo, plantas e outros materiais. $2^{\mathrm{a}}$.ed. Porto Alegre: Universidade Federal do Rio Grande do Sul; 1995.

Terekhova VA. Soil bioassay: Problems and approaches. Eurasian Soil Sci. 2011;44:173-9.

Tosza E, Dumnicka E, Niklińska M, Rożen A. Enchytraeid and earthworm communities along a pollution gradient near Olkusz (Southern Poland). Eurasian J Soil Biol. 2010;46:218-24.

United States Environmental Protection Agency - USEPA. Method 3051a: Microwave assisted acid digestion of sediments, sludges, soils, and oils. [internet]. EPA Publication; 2007. p.1-30. Disponível em: http://www.epa.gov/osw/hazard/testmethods/ sw846/pdfs/3051a.pdf. 